\title{
Prevalence, molecular characterization, and antibiotic susceptibility of Bacillus cereus isolated from dairy products in China
}

\author{
Shengjuan Zhao, ${ }^{1 *} \odot$ Junliang Chen, ${ }^{1 *} \odot$ Peng Fei, ${ }^{1} \odot$ Hongxia Feng, ${ }^{2}$ Yao Wang, ${ }^{1} \odot$ Md. Aslam Ali, ${ }^{3} \oplus$ \\ Shuzhen $\mathrm{Li}^{4,5}$ Haonan Jing, ${ }^{1}$ and Weiwei Yang ${ }^{6} \dagger{ }^{\circledR}$ \\ ${ }^{1}$ College of Food and Bioengineering, Henan University of Science and Technology, Luoyang 471023, China \\ ${ }^{2}$ Food and Pharmaceutical Engineering Institute, Guiyang University, Guiyang 550005, China \\ ${ }^{3}$ Department of Agro-Processing, Bangabandhu Sheikh Mujibur Rahman Agricultural University, Gazipur-1706, Bangladesh \\ ${ }^{4}$ Department of Immunology, College of Basic Medical Sciences, Shenyang Medical College, Shenyang 110034, China \\ ${ }^{5}$ Vascular Biology and Vascular Medicine Research Center, Shenyang Medical College, Shenyang 110034, China \\ ${ }^{6}$ The Department of Nutrition and Food Hygiene, Shenyang Medical College, Shenyang 110034, China
}

\section{ABSTRACT}

This study was conducted to reveal the prevalence, molecular characterization, and antibiotic susceptibility of Bacillus cereus isolated from dairy products including powdered infant formula, raw milk, pasteurized milk, ultra-high-temperature milk, and cheese. Five hundred samples collected from 5 provinces in China were analyzed in overall experiments. Multilocus sequence typing, distribution of toxin genes, and antibiotic susceptibility of the isolates were analyzed. Fifty-four $B$. cereus strains were detected; of these, 13 isolates (26\%) were from raw milk, 12 isolates $(12 \%)$ from pasteurized milk, 10 isolates (10\%) from cheese, 12 isolates (8\%) from ultra-high-temperature milk, and 7 isolates (7\%) from powdered infant formula. These isolates were divided into 24 sequence types (ST); among them, ST24, ST26, ST82, ST142, ST377, ST857, and ST1046 were the main dominant ST. The results of detection of toxin genes ( hblA, hblC, hblD, nheA, nheB, nheC, cytK, entFM, bceT, hlyII, and cesB) showed that $94.4 \%$ isolates carried nhe $A B C$ genes, whereas only $11.1 \%$ of the isolates contained the hblACD gene cluster. In addition, detection rates of $c y t K$, bceT, entFM, hlyII, and ces $B$ genes were $75.9,77.8,85.2,53.7$, and $11.1 \%$, respectively. The antibiotic susceptibility test indicated that most of $B$. cereus isolates were resistant to ampicillin, penicillin, cefepime, cephalothin, and oxacillin, and were susceptible to gentamicin, chloramphenicol, imipenem, tetracycline, ciprofloxacin, trimethoprimsulfamethoxazole, erythromycin, kanamycin, and cefotetan. Therefore, this study revealed the prevalence and

Received September 4, 2019.

Accepted December 17, 2019.

*These authors contributed equally to this work.

†Corresponding author: yw198269@163.com characteristics of $B$. cereus isolated from dairy products in China, indicating the potential risk and contributing to the effective prevention and control of this pathogen. Key words: Bacillus cereus, characterization, dairy product

\section{INTRODUCTION}

Bacillus cereus is a gram-positive, endospore-forming, foodborne pathogenic bacteria, and causes outbreaks of foodborne illness worldwide (Ehling-Schulz et al., 2012; Bennett et al., 2013). This pathogen can also cause gastric disease, emesis, diarrhea, and even death, and has been reported to have a high contamination rate in dairy products (Rosenquist et al., 2005; Kumari and Sarkar, 2014; Zhang et al., 2016). The high heat resistance of $B$. cereus strains increases their survival rates after heat treatment; meanwhile, stronger antibiotic resistance is generally considered to be a barrier against the effectiveness of antibiotics and disinfectants, which are probably the most important reasons why B. cereus has a higher contamination rate than other foodborne pathogens in dairy foods (Johnson et al., 1982). In recent studies (Kumari and Sarkar, 2014, 2016; Fei et al., 2019), the main concern of researchers is the harm and control of B. cereus in dairy products, whereas the contamination of this pathogen in dairy products has been ignored; therefore, it was necessary to conduct a systematic study on the prevalence and characteristics of B. cereus in dairy products in China.

Analysis of genetic diversity could improve the understanding of population characteristics of B. cereus, and facilitate establishment of accurate and rapid detection methods, reliable tracking of $B$. cereus, and the designation of targeted prevention and control measures (Park et al., 2009; Malek et al., 2013; Moradi-Khatoonabadi et al., 2014). Furthermore, the pathogenicity of $B$. cereus is associated with several toxins including he- 
molysin BL (Hbl), nonhemolytic enterotoxin (Nhe), cytotoxin K (CytK), enterotoxin FM (entFM), potential enterotoxins hemolysin II (HlyII), enterotoxin T (BceT), and emetic toxin (cereulide) that are usually produced by this pathogen and regulated by the ces gene (Fagerlund et al., 2004; Ehling-Schulz et al., 2006; Gao et al., 2018). Therefore, it is essential to detect these toxin genes to prevent and reduce the acute or chronic harmfulness of $B$. cereus strains in the dairy industry.

The use of antibiotics is still considered the most effective way to treat $B$. cereus infection in humans (Pena-Miller et al., 2013). Antibiotic-resistant B. cereus strains have been found due to the long-term unwise use of antibiotics or the emergence of resistant genes, resulting in horizontal gene transfer (Agers $\varnothing$ et al., 2002; Gao et al., 2018). In particular, the emergence of multidrug-resistant strains increases the chance of infection, resulting in a failure of antibiotic treatment (Ali et al., 2014). At present, the antibiotic resistance profile of $B$. cereus strains isolated from dairy products available in Chinese markets is not apparent. Hence, a relevant and fruitful investigation is necessary to increase the knowledge of the resistance status of $B$. cereus strains with a proposal of effective strategies to reduce the emergence of these strains.

The objective of this study was to investigate the contamination status of $B$. cereus isolates present in dairy products commonly available in Chinese markets and reveal the genotypes, toxin genes, antibiotic resistance, and biofilm formation of these isolates. A total of 54 $B$. cereus strains were isolated from 500 dairy products samples collected from locally available in Chinese markets from January 2018 to January 2019. Genotypes of the isolates were identified using multilocus sequence typing (MLST), and the toxin genes were detected using a PCR screening method. Antibiotic resistance of 54 B. cereus strains was also assessed. The data in this study would increase the ideas and knowledge about the pathogen $B$. cereus essentially present in dairy products and provide an important basis for the risk assessment of this pathogen.

\section{MATERIALS AND METHODS}

\section{Sample Collection}

A total of 500 dairy products samples were collected from January 2018 to January 2019, including 100 samples of powdered infant formula (PIF), 50 samples of raw milk (RM), 100 samples of pasteurized milk (PM), 150 samples of ultra-high-temperature milk (UHTM), and 100 samples of cheese. Among these samples, RM was collected from cattle farm and transferred to the sterile centrifuge tubes, then all samples were then stored in small coolers and quickly transported to the laboratory. Other samples were collected from commercially available dairy products and remained in their original commercial packaging, stored in small coolers before these samples arrived in the laboratory, and conserved at $4^{\circ} \mathrm{C}$ before isolation of $B$. cereus. These samples were collected from 5 provinces (Heilongjiang, Jilin, Hebei, Henan, and Guizhou) of northeast, central, and southwest China.

\section{Isolation and Identification of Bacterial Strains}

The isolation and identification of $B$. cereus were performed according to the method mentioned in National Food Safety Standard in China (GB4789. 14-2014; Ministry of Health of the People's Republic of China, 2014), with minor modifications. Twenty-five grams or $25 \mathrm{~mL}$ of sample was dissolved in $225 \mathrm{~mL}$ of brain-heart infusion broth followed by cultivation at $30 \pm 1^{\circ} \mathrm{C}$ for $24 \mathrm{~h}$. The cultures were streaked onto mannitol egg yolk polymyxin agar by adding mycosin $\mathrm{B}$ and yolk emulsion, and plates were incubated at $30^{\circ} \mathrm{C}$ for $24 \mathrm{~h}$. Typical pink colonies were picked up and inoculated into brain-heart infusion broth followed by propagation at $30 \pm 1^{\circ} \mathrm{C}$ for $24 \mathrm{~h}$. After propagation DNA was extracted from the culture and targeted strains were identified through $16 \mathrm{~S}$ rDNA sequencing.

\section{MLST Analysis}

The MSLT scheme was performed as described in a previous report (Zahner et al., 2013). Seven housekeeping genes ( $g l p F, g m k, i l v D, p t a, p u r, p y c A$, and tpi) were amplified and sequenced by the Beijing Genomics Institute (Beijing, China). The type sequences (ST) of $B$. cereus isolates were determined by genetic sequence alignment in the Bacillus cereus MLST database (http:/ /pubmlst.org/bcereus/). The phylogenetic relationship based on 2,829-bp sequences, concatenated sequences of 7 housekeeping genes, was analyzed using the maximum-likelihood algorithm in MEGA 6.0 (Arizona State University, Tempe), with 1,000 bootstrap replicates. Bacillus cereus ATCC 4342 and B. cereus 14579 were equally analyzed as reference strains.

\section{Detection of Toxin Genes}

Eleven toxin genes of $B$. cereus were detected using PCR screening, including 3 hemolytic enterotoxin genes $(h b l A, h b l C$, and $h b l D), 3$ nonhemolytic enterotoxin genes (nheA, nheB, and nheC), 4 other enterotoxigenic 
genes (cytK, bceT, entFM, and hlyII), and 1 cereulide synthetase gene $(c e s B)$. The PCR primer design and amplification strategies were performed according to the procedures as reported in previous studies (Hansen and Hendriksen, 2001; Fagerlund et al., 2004; Seong, 2008).

\section{Antibiotic Susceptibility Testing}

In accordance with the guidelines of the Clinical Laboratory Standards Institute (CLSI, 2015), the antibiotic resistance of 54 strains of $B$. cereus isolated from dairy products was assessed using the Kirby-Bauer disk diffusion method. Ampicillin $(10 \mu \mathrm{g})$, penicillin (10 U), cefepime $(30 \mu \mathrm{g})$, cephalothin $(30 \mu \mathrm{g})$, cefotetan $(30 \mu \mathrm{g})$, kanamycin $(30 \mu \mathrm{g})$, imipenem $(10 \mu \mathrm{g})$, gentamicin $(10 \mu \mathrm{g})$, erythromycin $(15 \mu \mathrm{g})$, oxacillin (1 $\mu \mathrm{g})$, ciprofloxacin $(5 \mu \mathrm{g})$, chloramphenicol $(30 \mu \mathrm{g})$, tetracycline $(30 \mu \mathrm{g})$, clindamycin $(2 \mu \mathrm{g})$, rifampin $(5 \mu \mathrm{g})$, and trimethoprim-sulfamethoxazole $(1.25 \mu \mathrm{g} / 23.75 \mu \mathrm{g})$ were used for the antibiotic susceptibility testing of $B$. cereus isolates. According to the diameter of inhibition zone, the antibiotic resistance of the isolates was interpreted as sensitive, intermediate, and resistant. Escherichia coli ATCC 25922 and Staphylococcus aureus ATCC 25923 were selected as the quality control organisms.

\section{RESULTS}

\section{Prevalence of B. cereus Isolated from Dairy Products in China}

As shown in Table 1, a total of 54 B. cereus strains were isolated from 500 dairy product samples with a contamination rate of $10.8 \%$. Of these samples, the contamination rate by $B$. cereus was the highest (13 isolates, 26\%) in RM samples followed by PM samples (12 isolates, $12 \%$ ), cheese samples (10 isolates, $10 \%$ ), UHTM samples (12 isolates, 8\%), and PIF samples (7 isolates, $7 \%$ ). In addition, among the 5 surveyed provinces, the highest contamination risk (12 isolates, 12\%) was observed in Guizhou, whereas the lowest contamination rate (9 isolates, 9\%) was in Hebei province.

\section{Genotyping Based on MLST}

Fifty-four strains of $B$. cereus isolates were divided into 24 ST; among them, ST24, ST26, ST82, ST142, ST377, ST857, and ST1046 were the main dominant ST (Table 2). Bacillus cereus ST82 was found in PIF, PM, and UHTM samples; meanwhile, B. cereus ST857 was detected in RM, PM, and UHTM samples. On the contrary, ST26, ST154, and ST392 were only found in cheese samples; ST43, ST205, and ST1341 were isolated from PM samples; and ST1336, and ST1968 appeared in only RM samples. An elaborated phylogenetic relationship of the 54 B. cereus strains (Figure 1) was clustered into 3 groups: group I (ST142, ST392, ST427, ST24, ST1968, ST154, ST66, ST857, ST1339, ST43, and ST1258), group II (ST82, ST125, ST377, and ST1098), and group III: (ST26, ST205, ST1046, ST1291, ST32, ST92, and ST1228). In addition, these results showed no obvious association between $B$. cereus genotype and collection regions.

\section{Distributions of Toxin Genes Among B. cereus Isolates}

The distributions of diarrheal enterotoxin genes (nhe$A B C$, hblACD, cytK, bceT, hly II, and entFM) and cesB associated with vomitus in $54 \mathrm{~B}$. cereus isolates obtained from dairy products are shown in Table 3 . Among diarrheal enterotoxin genes, detection rates of $h b l A, h b l C$, and $h b l D$ were $57.4,68.5$, and $16.7 \%$, respectively, and the $h b l A C D$ gene cluster was present in $11.1 \%$ of the $B$. cereus isolates. On the other hand, nhe $A B C$ genes were present in almost all isolates (94.4\%) and only $5.6 \%$ isolates did not have any of the nheA and nheB genes. In most of the isolates, cytK, bceT, entFM, and hlyII genes were found as $75.9,77.8,85.2$, and $53.7 \%$, respectively. In contrast, the cesB gene was detected in only $11.1 \%$ of the strains, which was significantly lower than any of the another 10 diarrheal enterotoxin genes.

Table 1. Prevalence and levels of Bacillus cereus isolated from dairy products in China

\begin{tabular}{|c|c|c|c|c|}
\hline Bacterial strain $^{1}$ & Source $^{2}$ & $\begin{array}{c}\text { No. of } \\
\text { samples }\end{array}$ & $\begin{array}{l}\text { No. of } \\
\text { B. cereus }\end{array}$ & $\begin{array}{c}\text { Contamination } \\
\text { rate }(\%)\end{array}$ \\
\hline HN1, GZ1, GZ2, JL1, HB1, HLJ1, HLJ2 & PIF & 100 & 7 & 7 \\
\hline HN2, HN3, HN4, GZ3, GZ4, GZ5, JL2, JL3, HLJ3, HLJ4, HLJ5, HB2, HB3 & $\mathrm{RM}$ & 50 & 13 & 26 \\
\hline HN7, HN8, HN9, GZ9, GZ10, JL7, JL8, JL9, HLJ8, HLJ9, HB6, HB7 & UHTM & 150 & 12 & 8 \\
\hline HN10, HN11, GZ11, GZ12, JL10, JL11, HLJ10, HLJ11, HB8, HB9 & Cheese & 100 & 10 & 10 \\
\hline Total & Dairy products & 500 & 54 & 10.8 \\
\hline
\end{tabular}

${ }^{1} \mathrm{HN}, \mathrm{GZ}, \mathrm{JL}, \mathrm{HB}$, and HLJ indicate that locations of isolation are Henan, Guizhou, Jilin, Hebei, and Heilongjiang provinces, respectively. ${ }^{2} \mathrm{PIF}=$ powdered infant formula; $\mathrm{RM}=$ raw milk; $\mathrm{PM}=$ pasteurized milk; UHTM $=$ ultra-high-temperature milk. 


\section{Antibiotic Resistance Profiles}

The antibiotic susceptibility of the $54 \mathrm{~B}$. cereus isolates to 17 antibiotics was tested and shown in Table 4. All isolates were susceptible to gentamicin and chloramphenicol, and most of the isolates were susceptible to imipenem $(98.15 \%)$, tetracycline $(98.15 \%)$, ciprofloxacin $(94.44 \%)$, trimethoprim-sulfamethoxazole $(85.18 \%)$, erythromycin $(83.33 \%)$, kanamycin $(83.33 \%)$, and cefotetan $(72.22 \%)$. On the contrary, most the B. cereus isolates were resistant to ampicillin (96.30\%), penicillin (96.30\%), rifampin $(96.30 \%)$, cefepime $(96.30 \%)$, oxacillin (90.74\%), and cephalothin (68.52\%). In addition, $83.33 \%$ of isolates were intermediate to clindamycin.

\section{DISCUSSION}

The ability of $B$. cereus to survive in dairy products was increased because of its strong tolerance against adverse environmental factors. Heini et al. (2018) reported that contamination rates of PIF in Switzerland were $78 \%$. The prevalence of $B$. cereus in the PIF Chinese retail market was also revealed by Zhang et al. (2017), who reported that $8.2 \%$ of PIF samples were contaminated with $B$. cereus strains. However, a more comprehensive understanding about the prevalence of $B$. cereus isolated from dairy products in China is still lacking. In this study, PIF, RM, PM, UHTM, and

Table 2. Multilocus sequence typing analysis of Bacillus cereus strains isolated from dairy products in China ${ }^{1}$

\begin{tabular}{|c|c|c|c|}
\hline $\mathrm{ST}$ & Bacterial strain & $\begin{array}{l}\text { No. (\%) } \\
\text { of isolates }\end{array}$ & Source \\
\hline $\mathrm{ST} 24$ & GZ11, GZ12, HN7 & $3 / 54(5.56)$ & Cheese, UHTM \\
\hline ST26 & JL10, HLJ10, HLJ11 & $3 / 54(5.56)$ & Cheese \\
\hline ST32 & GZ1, JL9 & $2 / 54(3.70)$ & PIF, UHTM \\
\hline ST43 & HB5 & $1 / 54(1.85)$ & $\mathrm{PM}$ \\
\hline ST66 & JL4, HB7 & $2 / 54(3.70)$ & PM, UHTM \\
\hline ST82 & HB1, HLJ7, HN9 & $3 / 54(5.56)$ & PIF, PM, UHTM \\
\hline ST92 & HN3, HLJ2 & $2 / 54(3.70)$ & PIF, RM \\
\hline ST125 & GZ4, JL7 & $2 / 54(3.70)$ & RM, UHTM \\
\hline ST142 & HN11, JL3, HLJ4 & $3 / 54(5.56)$ & Cheese, RM \\
\hline ST154 & HB8, JL11 & $2 / 54(3.70)$ & Cheese \\
\hline ST205 & GZ6, GZ8 & $2 / 54(3.70)$ & $\mathrm{PM}$ \\
\hline ST377 & GZ2, HB3, GZ10 & $3 / 54(5.56)$ & PIF, RM, UHTM \\
\hline ST392 & HN10, HB9 & $2 / 54(3.70)$ & Cheese \\
\hline ST427 & HN1, HLJ8 & $2 / 54(3.70)$ & PIF, UHTM \\
\hline ST857 & GZ5, JL5, HLJ9 & $3 / 54(5.56)$ & RM, PM, UHTM \\
\hline ST1046 & HLJ1, JL1, JL2 & $3 / 54(5.56)$ & PIF, RM \\
\hline ST1098 & HLJ3, GZ7 & $2 / 54(3.70)$ & $\mathrm{RM}, \mathrm{PM}$ \\
\hline ST1228 & HN5, HN8 & $2 / 54(3.70)$ & PM, UHTM \\
\hline ST1258 & HN2, GZ9 & $2 / 54(3.70)$ & RM, UHTM \\
\hline ST1291 & HN6, JL8 & $2 / 54(3.70)$ & PM, UHTM \\
\hline ST1336 & GZ3, HB4 & $2 / 54(3.70)$ & $\mathrm{RM}$ \\
\hline ST1339 & HLJ5, HB6 & $2 / 54(3.70)$ & RM, UHTM \\
\hline ST1341 & JL6, HLJ6 & $2 / 54(3.70)$ & $\mathrm{PM}$ \\
\hline ST1968 & HN4, HB2 & $2 / 54(3.70)$ & $\mathrm{RM}$ \\
\hline
\end{tabular}

${ }^{1} \mathrm{ST}=$ sequence type $\mathrm{PIF}=$ powdered infant formula $; \mathrm{RM}=$ raw milk; $\mathrm{PM}=$ pasteurized milk; UHTM = ultra-high-temperature milk. cheese were collected as research objects, and the contamination rates of the above-mentioned dairy products were $7,26,12,8$, and $10 \%$, respectively, indicating the potential risk of $B$. cereus in dairy products of China.

In comparison to previous studies, genetic typing based on the MLST method is more conducive to revealing the molecular characteristics of the tested strains and contributing to the traceability of pathogenic bacteria (Fei et al., 2015). The 54 strains of B. cereus in this study were divided into 24 ST using MLST, indicating that $B$. cereus isolated from dairy products had a high genetic diversity. Among these, ST26 was found in rice flour, PIF, cooked rice, fried rice noodle, hot dog, and cold noodle samples (Yang et al., 2017a,b), which indicates that ST26 is likely to be the most predominant ST of $B$. cereus isolated from food samples. In the study of Zhang et al. (2017), B. cereus ST32 and ST142 strains were isolated from infant formula samples; similarly, our results showed that ST32 was found in PIF and UHTM samples, and ST142 was detected in cheese and RM samples. The strains of $B$. cereus ST92, ST205, ST427, and ST857 were detected in dairy products at varying degrees; interestingly, these ST were also found on the surfaces of equipment associated with processing of the UHT milk (Lin et al., 2017). Therefore, the processing equipment as well as environments might be the possible sources of $B$. cereus strains in dairy products.

The distribution and proportions of 10 toxin genes were revealed in this study. Toxins such as Hbl, Nhe, and CytK had been reported as the major etiology of diarrhea caused by food poisoning (Lindbäck et al., 2004; Ngamwongsatit et al., 2008). The presence of $\mathrm{Hbl}$ and Nhe toxins could be ascertained only with the detection of toxin genes $h b l A C D$ and nhe $A B C$, respectively. In this study, the $h b l A C D$ gene cluster was found in $11.1 \%$ of the B. cereus strains (Table 3 ) isolated from dairy products, which is significantly different from the previous studies (Cui et al., 2016; Yang et al., 2017a; Zhang et al., 2017). According to the reports of Yang et al. (2017b) and Zhang et al. (2017), no hblACD gene cluster was found in $B$. cereus isolated from infant formula in China, and B. cereus with hblACD gene cluster is not common in food from China. However, a higher detection rate $(78.3 \%)$ of hblACD gene cluster was found in $B$. cereus strains of milk origin (Cui et al., 2016). The proportions of nhe $A B C$ gene cluster and CytK in B. cereus in our study were 94.4 and $75.9 \%$, respectively, which are approximately similar to the detection rates (93 and $73 \%$, respectively) of the abovementioned genes in B. cereus isolated from PM (Gao et al., 2018). Besides, cesB associated with vomitus was present in $11.1 \%$ of B. cereus strains (Table 3 ) isolated from dairy products, which is lower than that from food 
and clinical samples (100\%) and UHT milk processing lines (48.15\%; Lin et al., 2017; Yang et al., 2017a) but higher than that isolated from PM $(5 \%)$ in China (Cui et al., 2016). Therefore, the outcomes of this study describe that $11.1 \%$ of the isolates, containing both diarrhea and vomit virulence, might induce a high risk in serious infections by $B$. cereus that would essentially be isolated from various dairy products available in Chinese markets.

The antibiotic susceptibility tests showed that most of $B$. cereus strains isolated from dairy products were resistant to $\beta$-lactam antibiotics including ampicillin, penicillin, cefepime, cephalothin, and oxacillin, which is consistent with previous studies on the antibiotic resis-

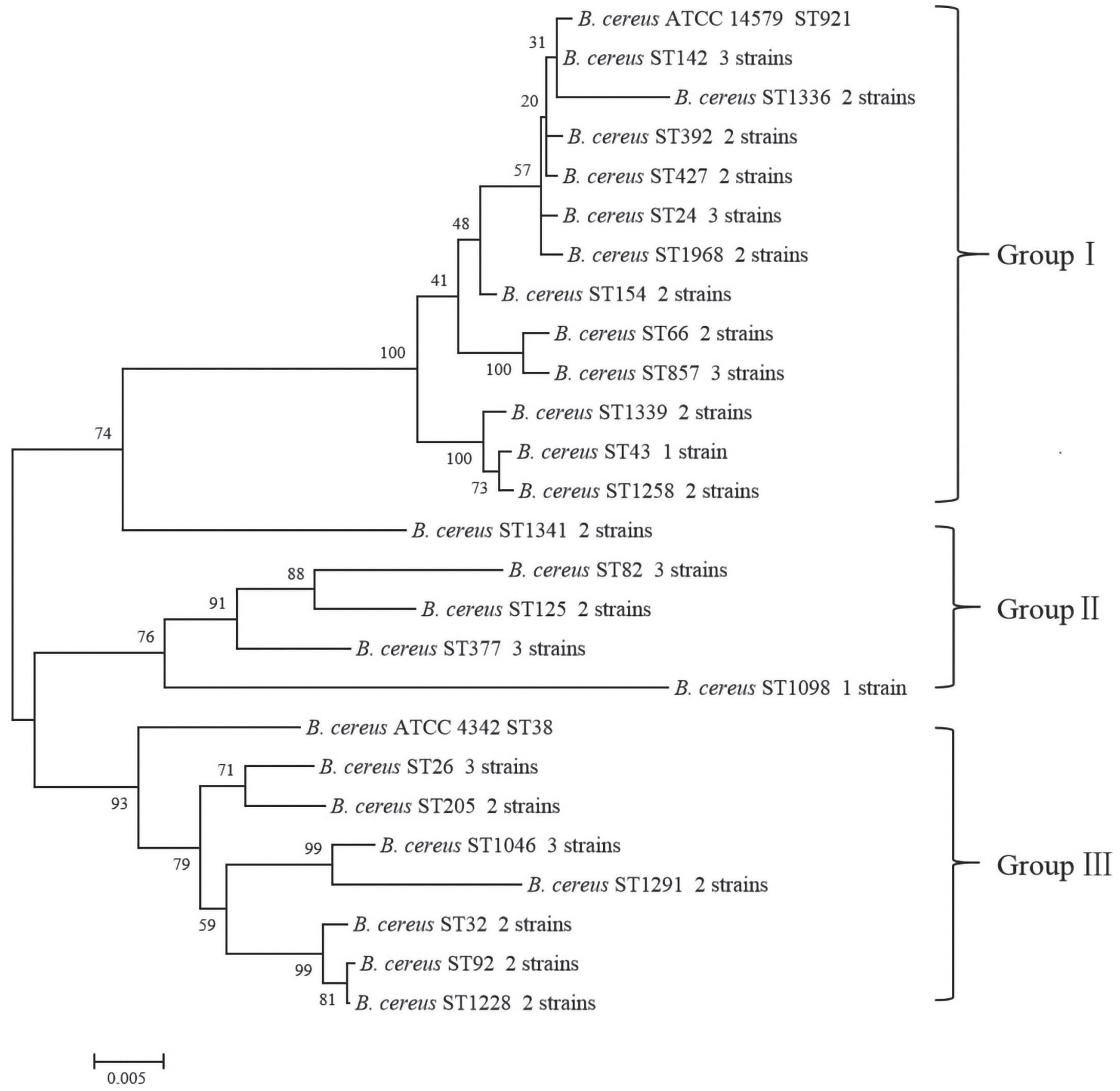

Figure 1. Maximum-likelihood tree of multilocus sequence typing 7 loci ( $g l p F$, gmk, ilvD, pta, pur, pycA, and tpi) of Bacillus cereus strains isolated from powdered food products in China. Bacillus cereus ATCC 4342 and B. cereus 14579 were equally analyzed as reference strains. The tree was generated using MEGA 6.0 (Arizona State University, Tempe) with 1,000 bootstrap replicates. ST = sequence type. 
Table 3. Distributions of toxin genes among Bacillus cereus strains isolated from dairy products in China $^{1}$

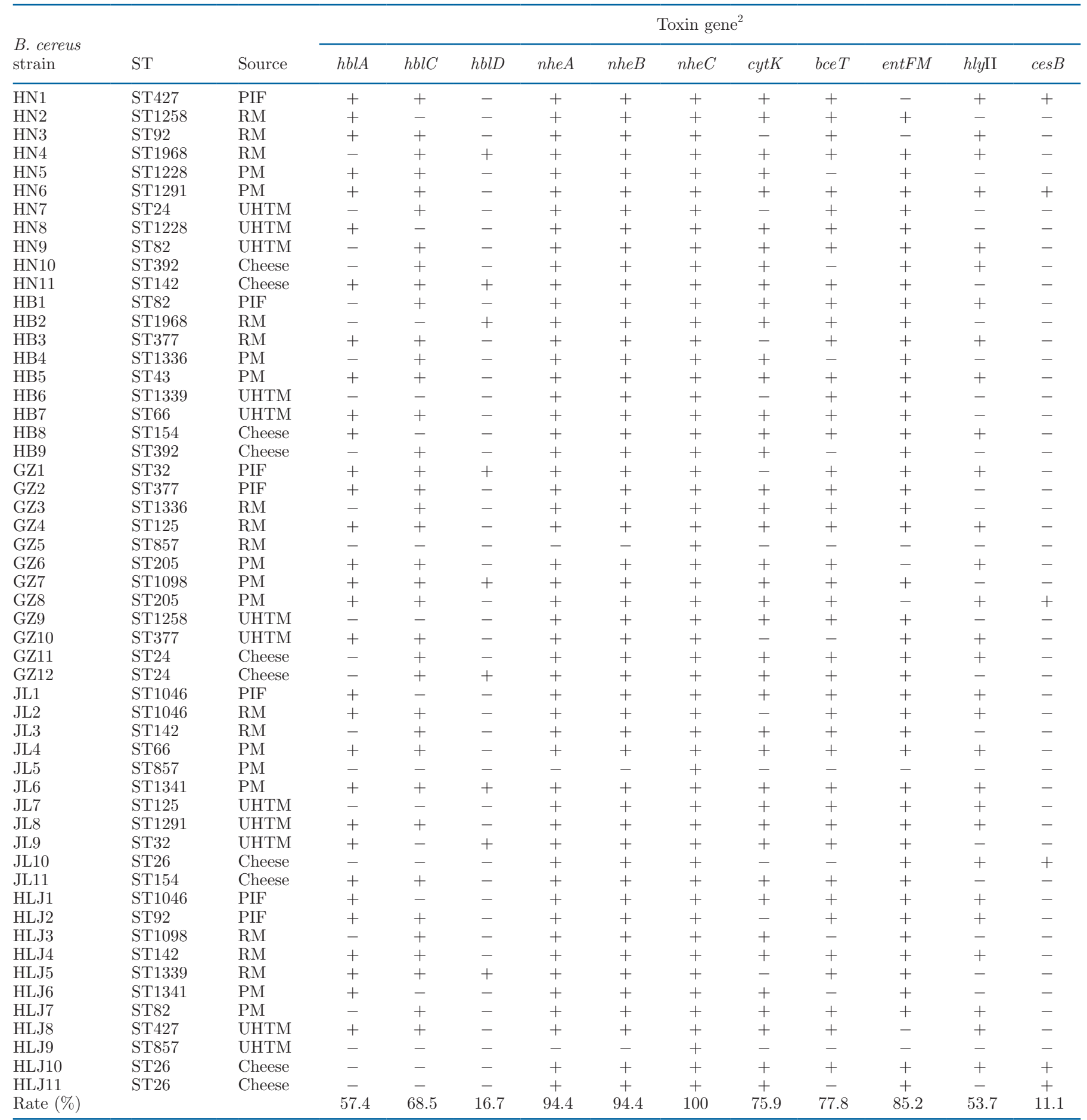

${ }^{1} \mathrm{ST}=$ sequence type; PIF $=$ powdered infant formula; $\mathrm{RM}=$ raw milk; PM = pasteurized milk; UHTM = ultra-high-temperature milk. ${ }^{2}+$ indicates a positive result, and - indicates a negative result.

tance of B. cereus in fermented soybean products, PM, and cheese (Yim et al., 2015; Yibar et al., 2017; Gao et al., 2018). Meanwhile, the antibiotic resistance profiles of 54 isolates to ciprofloxacin, kanamycin, gentamicin, erythromycin, chloramphenicol, clindamycin, rifampin, and trimethoprim-sulfamethoxazole were similar to that of this pathogen isolated from PM in China (Park et al., 2009; Gao et al., 2018). In addition, all of the 54 
Table 4. Antibiotic susceptibility of 54 Bacillus cereus strains isolated from dairy products in China

\begin{tabular}{lccc}
\hline & \multicolumn{3}{c}{ B. cereus $(\mathrm{n}=54)^{1}$} \\
\cline { 2 - 4 } Antimicrobial agent & No. $(\%)$ of S & No. (\%) of I & No. (\%) of R \\
\hline Ampicillin & $1(1.85)$ & $1(1.85)$ & $52(96.30)$ \\
Penicillin & $2(3.70)$ & $0(0)$ & $52(96.30)$ \\
Cefepime & $2(3.70)$ & $0(0)$ & $52(96.30)$ \\
Cephalothin & $5(9.25)$ & $12(22.22)$ & $37(68.52)$ \\
Cefotetan & $39(72.22)$ & $7(12.96)$ & $8(14.81)$ \\
Kanamycin & $45(83.33)$ & $8(14.81)$ & $1(1.85)$ \\
Imipenem & $53(98.15)$ & $1(1.85)$ & $0(0)$ \\
Gentamicin & $54(100)$ & $0(0)$ & $0(0)$ \\
Erythromycin & $45(83.33)$ & $7(12.96)$ & $2(3.70)$ \\
Oxacillin & $2(3.70)$ & $3(5.56)$ & $49(90.74)$ \\
Ciprofloxacin & $51(94.44)$ & $3(5.56)$ & $0(0)$ \\
Chloramphenicol & $54(100)$ & $0(0)$ & $0(0)$ \\
Tetracycline & $53(98.15)$ & $1(1.85)$ & $0(0)$ \\
Clindamycin & $4(7.41)$ & $45(83.33)$ & $5(9.26)$ \\
Rifampin & $0(0)$ & $2(3.70)$ & $52(96.30)$ \\
Trimethoprim-sulfamethoxazole & $46(85.18)$ & $4(7.41)$ & $4(7.41)$ \\
\hline
\end{tabular}

${ }^{1}$ After the treatments with antibiotics of recommended concentration, if the diameter of inhibition zones against B. cereus was greater than that of quality control organisms, the effect of antibiotics on B. cereus was defined as $\mathrm{S}$; if the diameter of inhibition zones against $B$. cereus was less than that of quality control organisms, the effect of antibiotics on B. cereus was defined as $\mathrm{R}$; and if the diameter was between $\mathrm{R}$ and $\mathrm{S}$, the effect of antibiotics on B. cereus was defined as I.

strains of $B$. cereus were susceptible to gentamicin and chloramphenicol, which suggested that these 2 antibiotics could be used for treatment against the gastrointestinal diseases caused by $B$. cereus infections.

\section{CONCLUSIONS}

The present study revealed the contamination condition of B. cereus in dairy products available in China. The MLST analysis indicated that high genetic diversity is present in $B$. cereus isolated from dairy products in China, and ST24, ST26, ST82, ST142, ST377, ST857, and ST1046 were the main dominant ST. The nheABC genes were present in almost all isolates, whereas the cesB gene was detected in only $11.1 \%$ of the strains. In addition, compared with the previous monitoring, the antibiotic resistance of $B$. cereus isolated from dairy products in China has not increased significantly, and gentamicin and chloramphenicol are better used to prevent and control this pathogen. These results enhance the perception about contamination and characteristic outcomes of $B$. cereus strains isolated from different dairy products abundantly available in Chinese markets, and provide a theoretical basis for developing the effective measures to reduce the contamination of this pathogen.

\section{ACKNOWLEDGMENTS}

This study was supported by the Doctor Scientific Research Start-up Fund of Henan University of Sci- ence and Technology (13480068, Luoyang, China), Science and Technology Project of Henan Province (172102110019, Zhengzhou, China), and National Natural Science Foundation of China (31702218 and 31601450, Beijing, China). All authors have stated that there are no conflicts of interest.

\section{REFERENCES}

Agers $\varnothing$, Y., L. B. Jensen, M. Givskov, and M. C. Roberts. 2002. The identification of a tetracycline resistance gene tet(M), on a Tn916like transposon, in the Bacillus cereus group. FEMS Microbiol. Lett. 214:251-256. https://doi.org/10.1016/S0378-1097(02)00883 -2 .

Ali, M. M. M., A. H. Aburowes, A. M. Albakush, M. M. Rzeg, A. Alrtail, and K. S. Ghenghesh. 2014. Identification of multidrugresistant bacteria and Bacillus cereus from healthcare workers and environmental surfaces in a hospital. Libyan J. Med. 9:25794. https://doi.org/10.3402/ljm.v9.25794.

Bennett, S. D., K. A. Walsh, and L. H. Gould. 2013. Foodborne disease outbreaks caused by Bacillus cereus, Clostridium perfringens, and Staphylococcus aureus-United States, 1998-2008. Clin. Infect. Dis. 57:425-433. https://doi.org/10.1093/cid/cit244.

CLSI. 2015. "Disk difffusion," in performance standards for antimicrobial susceptibility testing; 15th Informational Supplement, Vol 35. CLSI, Wayne, PA.

Cui, Y., Y. Liu, X. Liu, X. Xia, S. Ding, and K. Zhu. 2016. Evaluation of the toxicity and toxicokinetics of cereulide from an emetic Bacillus cereus strain of milk origin. Toxins (Basel) 8:156. https:/ /doi.org/10.3390/toxins8060156.

Ehling-Schulz, M., M. Guinebretiere, A. Monthán, O. Berge, M. Fricker, and B. Svensson. 2006. Toxin gene profiling of enterotoxic and emetic Bacillus cereus. FEMS Microbiol. Lett. 260:232-240. https: //doi.org/10.1111/j.1574-6968.2006.00320.x.

Ehling-Schulz, M., U. Messelhäusser, and J. Hoorfar. 2012. One pathogen but two different types of foodborne outbreak: Bacillus cereus in catering facilities in Germany. Case Studies in Food Safety and Authenticity 63-70. 
Fagerlund, A., O. Ween, T. Lund, S. P. Hardy, and P. E. Granum. 2004. Genetic and functional analysis of the cytK family of genes in Bacillus cereus. Microbiology 150:2689-2697. https://doi.org/10 $.1099 /$ mic.0.26975-0.

Fei, P., C. Man, B. Lou, S. J. Forsythe, Y. Chai, R. Li, J. Niu, and Y. Jiang. 2015. Genotyping and source tracking of Cronobacter sakazakii and C. malonaticus isolates from powdered infant formula and an infant formula production factory in China. Appl. Environ. Microbiol. 81:5430-5439. https://doi.org/10.1128/AEM.01390-15.

Fei, P., Y. Xu, S. Zhao, S. Gong, and L. Guo. 2019. Olive oil polyphenol extract inhibits vegetative cells of Bacillus cereus isolated from raw milk. J. Dairy Sci. 102:3894-3902. https://doi.org/10.3168/ jds.2018-15184.

Gao, T., Y. Ding, Q. Wu, J. Wang, J. Zhang, S. Yu, P. Yu, C. Liu, L. Kong, Z. Feng, M. Chen, S. Wu, H. Zeng, and H. Wu. 2018 Prevalence, virulence genes, antimicrobial susceptibility, and genetic diversity of Bacillus cereus isolated from pasteurized milk in China. Front. Microbiol. 9:533. https://doi.org/10.3389/fmicb .2018.00533.

Hansen, B. M., and N. B. Hendriksen. 2001. Detection of enterotoxic Bacillus cereus and Bacillus thuringiensis strains by PCR analysis. Appl. Environ. Microbiol. 67:185-189. https://doi.org/10.1128/ AEM.67.1.185-189.2001.

Heini, N., R. Stephan, M. Ehling-Schulz, and S. Johler. 2018. Characterization of Bacillus cereus group isolates from powdered food products. Int. J. Food Microbiol. 283:59-64. https://doi.org/10 .1016/j.ijfoodmicro.2018.06.019.

Johnson, K. M., C. L. Nelson, and F. F. Busta. 1982. Germination and heat resistance of Bacillus cereus spores from strains associated with diarrheal and emetic food-borne illnesses. J. Food Sci. 47:1268-1271. https://doi.org/10.1111/j.1365-2621.1982.tb07663 .x.

Kumari, S., and P. K. Sarkar. 2014. Prevalence and characterization of Bacillus cereus group from various marketed dairy products in India. Dairy Sci. Technol. 94:483-497. https://doi.org/10.1007/ s13594-014-0174-5.

Kumari, S., and P. K. Sarkar. 2016. Bacillus cereus hazard and control in industrial dairy processing environment. Food Control 69:20-29. https://doi.org/10.1016/j.foodcont.2016.04.012.

Lin, Y., F. Ren, L. Zhao, and H. Guo. 2017. Genotypes and the persistence survival phenotypes of Bacillus cereus isolated from UHT milk processing lines. Food Control 82:48-56. https://doi.org/10 .1016/j.foodcont.2017.06.025.

Lindbäck, T., A. Fagerlund, M. S. Rødland, and P. E. Granum. 2004. Characterization of the Bacillus cereus Nhe enterotoxin. Microbiology 150:3959-3967. https://doi.org/10.1099/mic.0.27359-0.

Malek, F., B. M. Boudjemaa, A. Aouar-Métri, and M. Kihal. 2013. Identification and genetic diversity of Bacillus cereus strains isolated from a pasteurized milk processing line in Algeria. Dairy Sci. Technol. 93:73-82. https://doi.org/10.1007/s13594-012-0093-2.

Ministry of Health of the People's Republic of China. 2014. GB 4789.14-2014. National Food Safety Standard: Food Microbiological Examination: Bacillus cereus. China Standard Press, Beijing.

Moradi-Khatoonabadi, Z., H. Ezzatpanah, Y. Maghsoudlou, M. Khomeiri, and M. Aminafshar. 2014. Tracking Bacillus cereus in UFfeta cheese processing line. Int. Dairy J. 39:47-52. https://doi.org/ 10.1016/j.idairyj.2014.04.009.

Ngamwongsatit, P., W. Buasri, P. Pianariyanon, C. Pulsrikarn, M. Ohba, A. Assavanig, and W. Panbangred. 2008. Broad distribution of enterotoxin genes (hblCDA, nheABC, cytK, and entFM) among Bacillus thuringiensis and Bacillus cereus as shown by novel primers. Int. J. Food Microbiol. 121:352-356. https://doi.org/10.1016/ j.ijfoodmicro.2007.11.013.
Park, Y. B., J. B. Kim, S. W. Shin, J. C. Kim, S. H. Cho, B. K. Lee, J. Ahn, J. M. Kim, and D. H. Oh. 2009. Prevalence, genetic diversity, and antibiotic susceptibility of Bacillus cereus strains isolated from rice and cereals collected in Korea. J. Food Prot. 72:612-617. https://doi.org/10.4315/0362-028X-72.3.612.

Pena-Miller, R., D. Laehnemann, G. Jansen, A. Fuentes-Hernandez, P. Rosenstiel, H. Schulenburg, and R. Beardmore. 2013. When the most potent combination of antibiotics selects for the greatest bacterial load: The smile-frown transition. PLoS Biol. 11:e1001540. https://doi.org/10.1371/journal.pbio.1001540.

Rosenquist, H. L. Smidt, S. R. Andersen, G. B. Jensen, and A. Wilcks. 2005. Occurrence and significance of Bacillus cereus and Bacillus thuringiensis in ready-to-eat food. FEMS Microbiol. Lett. 250:129-136. https://doi.org/10.1016/j.femsle.2005.06.054.

Seong, S. J. 2008. Toxin gene profiling of Bacillus cereus food isolates by PCR. J. Korean Soc. Appl. Biol. Chem. 51:263-268.

Yang, Y., H. Gu, X. Yu, L. Zhan, J. Chen, Y. Luo, Y. Zhang, Y. Zhang, Y. Lu, J. Jiang, and L. Mei. 2017a. Genotypic heterogeneity of emetic toxin producing Bacillus cereus isolates from China. FEMS Microbiol. Lett. 364:fnw237. https://doi.org/10.1093/ femsle/fnw237.

Yang, Y., X. Yu, L. Zhan, J. Chen, Y. Zhang, J. Zhang, H. Chen, Z. Zhang, Y. Zhang, Y. Lu, and L. Mei. 2017b. Multilocus sequence type profiles of Bacillus cereus isolates from infant formula in China. Food Microbiol. 62:46-50. https://doi.org/10.1016/j.fm 2016.09.007.

Yibar, A., F. Cetinkaya, E. Soyutemiz, and G. Yaman. 2017. Prevalence, enterotoxin production and antibiotic resistance of Bacillus cereus isolated from milk and cheese. Kafkas Univ. Vet. Fak. 23:635-642.

Yim, J. H., K. Y. Kim, J. W. Chon, D. H. Kim, H. S. Kim, D. S. Choi, I. S. Choi, and K. H. Seo. 2015. Incidence, antibiotic susceptibility, and toxin profiles of Bacillus cereus sensu lato isolated from Korean fermented soybean products. J. Food Sci. 80:1266-1270. https://doi.org/10.1111/1750-3841.12872.

Zahner, V., A. C. Silva, G. P. Moraes, D. Mcintosh, and I. Filippis. 2013. Extended genetic analysis of Brazilian isolates of Bacillus cereus and Bacillus thuringiensis. Mem. Inst. Oswaldo Cruz 108:6572. https://doi.org/10.1590/S0074-02762013000100011.

Zhang, Y., J. Chen, C. Feng, L. Zhan, J. Zhang, Y. Li, Y. Yang, H. Chen, Z. Zhang, Y. Zhang, L. Mei, and H. Li. 2017. Quantitative prevalence, phenotypic and genotypic characteristics of Bacillus cereus isolated from retail infant foods in China. Foodborne Pathog. Dis. 14:564-572. https://doi.org/10.1089/fpd.2017.2287.

Zhang, Z., L. Feng, H. Xu, C. Liu, N. P. Shah, and H. Wei. 2016. Detection of viable enterotoxin-producing Bacillus cereus and analysis of toxigenicity from ready-to-eat foods and infant formula milk powder by multiplex PCR. J. Dairy Sci. 99:1047-1055. https://doi .org/10.3168/jds.2015-10147.

\section{ORCIDS}

Shengjuan Zhao @ https://orcid.org/0000-0002-8647-0470 Junliang Chen @ https://orcid.org/0000-0001-9452-4697

Peng Fei @ https://orcid.org/0000-0001-7895-4603

Yao Wang $\odot$ https://orcid.org/0000-0002-7193-3276

Md. Aslam Ali $\odot$ https://orcid.org/0000-0002-6382-2787

Weiwei Yang ๑ https://orcid.org/0000-0001-8286-8708 